\title{
Development of a Fast Marx Generator for High Power Ultra Wideband Pulse Radiation
}

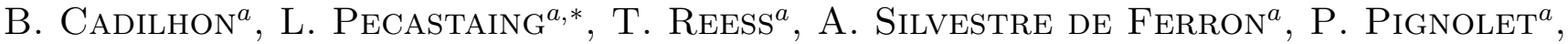

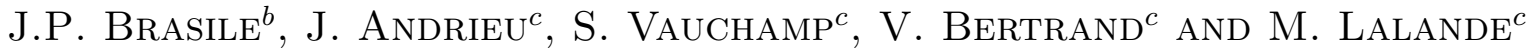 \\ ${ }^{a}$ Laboratoire de Génie Electrique, Université de Pau, 2 av. Angot, 64053 Pau, France \\ ${ }^{b}$ Thales Communications SA, 160 boulevard de Valmy, 92704 Colombes, France \\ ${ }^{c}$ XLIM/OSA, IUT GEII, 7 rue Jules Vallès, 19100 Brive, France
}

\begin{abstract}
We present in this paper the design and realisation of two autonomous, ultra wideband radiation sources consisting of a high gain broadband antenna driven by two subnanosecond pulsed power sources. Each one is made of a Marx generator and a pulse forming device based on the use of gaseous spark gap. The main pulsed source is a ten stage subnanosecond Marx generator which delivers pulses in the range of $250 \mathrm{kV} / 1.5 \mathrm{~J}$, with a $300 \mathrm{ps}$ rise time, subnanosecond pulse duration at a pulse repetition frequency of $350 \mathrm{~Hz}$. The antenna combined with the pulsed source is a travelling wave antenna called Valentine antenna. Some mechanical modifications were made to improve the dielectric strength of this radiation element. A 3D model of the antenna, on a time domain electromagnetic software, was first performed to study the influence of these modifications on the main radiating characteristics of the antenna. Its high gain and its capability to radiate short pulses without dispersion allow us to achieve a high measured figure of merit.
\end{abstract}

PACS numbers: 84.70.+p, 07.57.Hm, 84.30.Ng

\section{Introduction}

Recently, there has been considerable interest in high power microwave (HPM) sources for many military and civilian applications, such as target identification, detection of buried targets, humanitarian demining, nonlethal directed energy weaponry and also susceptibility and hardening tests on electronics systems. The production of high power electromagnetic fields, in this way, seems to be a real application for high pulsed power devices. These systems, consisting of an electrical pulsed power source and an antenna, provide a radiated electromagnetic environment with a fairly flat spectral content over one to two decades. Nowadays, our work is currently focused on a new project concerning an autonomous ultra wideband (UWB) pulser for the electromagnetic radiation [1]. We studied the feasibility of a whole electrical solution for the design of a compact and autonomous high-voltage subnanosecond pulsed source. The electrical energy delivered is transported by a TEM wave and converted into the electromagnetic radiation energy with the use of a convenient high voltage ultra-wideband (HV UWB) antenna.

\section{Generation of high power subnanosecond pulse}

A main device has been designed to achieve higher output voltages and higher repetition rates. This pulsed source is made of a fast Marx generator in which a pulse forming device is directly inserted on the latest stage.

* corresponding author; e-mail: laurent.pecastaing@univ-pau.fr
The Marx generator is made of ten stages vertically piled up in a cylindrical metallic tight enclosure of $20 \mathrm{dm}^{3}$. Each stage is composed of a $500 \mathrm{pF} / 100 \mathrm{kV}$ DC ceramic capacitor, resistively charged by a $33 \mathrm{k} \Omega$ carbon resistor, and a set of two steel electrodes. The special assembly of the stages on two columns and the special layout of discharging resistors $[1,2]$ reduce the charging time of the capacitors and thus enhance the pulse repetition frequency achievable by the generator. Theoretically, the generator could erect at a frequency of $435 \mathrm{~Hz}$ without decreasing of the output voltage amplitude [3].

The pulse forming device is connected to the last stage of the generator. It consists also of a peaking capacitor and a crowbar gap used to cut the exponential fall of the pulse. For a well known spark gap spacing and values of pressure higher than that of the minimum of the Paschen law, the gas breakdown voltage, in a uniform electric field, increases in direct ratio to the pressure of the gas in the spark gap. Several tests in air and air-SF 6 mixtures were investigated to characterize the output pulse in amplitude and rise time. A linear variation of the pulse amplitude with the pressure is observed in Fig. 1. But for mechanical reasons, the air-SF ${ }_{6}$ mixture is preferred to reach higher voltage levels without damaging the rise time.

The distance of the crowbar gap is fixed to $1.5 \mathrm{~mm}$. It seems to be the best compromise between efficiency and pulse width. We present, in Fig. 2, a typical pulse on a $50 \Omega$ load measured by a $2.3 \mathrm{GHz}$ capacitive line divider. The amplitude is $226 \mathrm{kV}$, the rise time is $360 \mathrm{ps}$ and the pulse width is $410 \mathrm{ps}$ which represents a peak power of $1 \mathrm{GW}$.

Repetitive investigations have been carried out and a pulse repetition frequency of $350 \mathrm{~Hz}$ has been reached. 


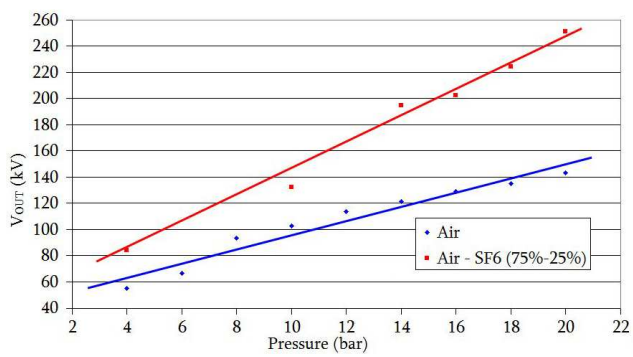

Fig. 1. Output voltage versus pressure in air and $75 \%$ air-25\% $\mathrm{SF}_{6}$ mixture without crowbar sbarkgap.

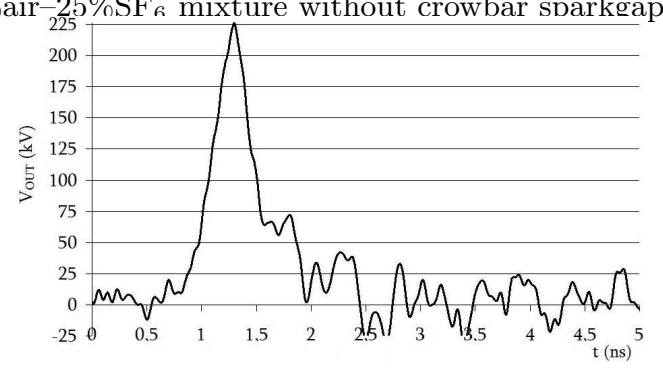

Fig. 2. Output waveform on a $50 \Omega$ load.

For the moment this value is limited by the ability of the power supply to load the Marx capacitors after each discharge.

\section{Experimental investigations}

Because of its high gain and its capability to radiate short pulses without dispersion in the frequency band $300 \mathrm{MHz}-2.5 \mathrm{GHz}$, the Valentine antenna [4, 5] was chosen to be the radiating element of high-power UWB sources. A mechanical matching of the antenna, using the time-domain electromagnetic software CST Microwave Studio, was done to improve its dielectric strength. This antenna is originally composed of two metallic strips curved along a specific profile and a $50 \Omega$ coaxial-to-stripline transition at the input. This structure is supported by two wood sides. Thus, this whole set is $1200 \mathrm{~mm}$ high, $1000 \mathrm{~mm}$ long, $400 \mathrm{~mm}$ wide. The design of these modifications, its shape and its influence on the electromagnetic characteristics of the antenna were studied in order not to disturb the electromagnetic characteristics of the radiating element.

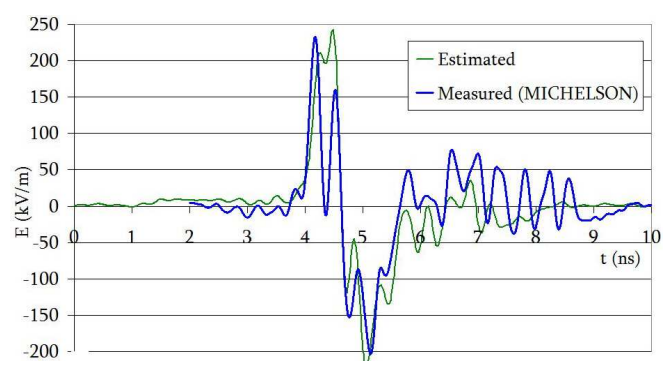

Fig. 3. Waveform of the radiated pulse.
A new HV Valentine antenna is combined with the pulsed source through a $50 \Omega$ coaxial transmission line in which a capacitive divider is inserted. The recorded pulse permits to estimate, through a frequency calculation, the estimated field and thus the figure of merit.

A new method to measure very intensive electromagnetic fields using the target diffraction of the incident field is developed [6]. The diffracted field is measured by a classical low power UWB antenna. The target used has small dimensions and no cables are used in the field region thus the electromagnetic perturbations generated and undergone by the measurement device are considerably limited. This method is applied to measure the high-power pulse delivered by the second pulsed source. The figure of merit measured is $436 \mathrm{kV}$ which is close to the estimated result (Fig. 3).

\section{Conclusion}

A $1 \mathrm{GW}$ peak power source was developed based on the combination of a Marx generator, a pulse forming device and a travelling wave antenna. It produces pulses in the subnanosecond range, with hundreds of picoseconds rise time and subnanosecond pulse width. Repetition rate of several hundreds $\mathrm{Hz}$ are achievable. By means of an electromagnetic simulation software, the Valentine antenna has been fitted to high-voltage, high-power applications. A $50 \Omega$ oil insulated transition has been specially designed to realize a geometrical adaptation between the coaxial feed line and the strip line structure of the antenna.

The figure-of-merit measured is $436 \mathrm{kV}$ for the complete pulsed source. We are currently working on the improvement of the $\mathrm{HV}$ pulsed source to reach output voltage in the range of $500 \mathrm{kV}$ expecting to reach high figure of merit.

\section{References}

[1] B. Cadilhon, Ph.D. Thesis, Pau University, France, 2008, p. 274.

[2] B. Cadilhon, L. Pécastaing, T. Reess, A. Gibert, IET Elect. Power Appl. 2, 4 (2008).

[3] G.A. Dawson, D.E. Davies, Research Notes, Br. J. Appl. Phys. 14, 155 (1963).

[4] J.-C. Diot, P. Delmote, J. Andrieu, M. Lalande, V. Bertrand, B. Jecko, S. Colson, R. Guillerey, M. Brishoual, IEEE Trans. Antennas Propag. 55, 987 (2007).

[5] P.R. Foster, S.M. Tun, Proc. Ninth Intern. Conf. Antennas Propagation, 1995, p. 286.

[6] S. Vauchamp, M. Lalande, J. Andrieu, B. Jecko, J.L. Lasserre, A. Paupert, G. Teyssidou, R. Pouzalgues, Proc. Intern. Conf. Electromagnetics in Advanced Applications (ICEAA), Turin 2007. 